\title{
ARTÍCULOS
}

Recibido 08.01.2014. Aprobado 27.05.2014

Evaluado por el sistema double blind review. Editora cientifica: María Antonia García Benau

DOI: http://dx.doi.org/10.1590/So034-759020150308

\section{PRÁCTICAS DE RESPONSABILIDAD SOCIAL, REPUTACIÓN CORPORATIVA Y DESEMPEÑO FINANCIERO}

\author{
Práticas de responsabilidade social, reputação corporativa e desempenho \\ financeiro
}

Social responsability practices, corporate reputation and financial performance

\section{RESUMEN}

Este trabajo analiza si la adopción de estrategias de divulgación de información voluntaria de prácticas de Responsabilidad Social Empresarial (RSE) a través de distintos medios de comunicación tiene incidencia sobre el desempeño financiero y la reputación corporativa. Una vez identificadas las distintas dimensiones por las cuales las empresas emiten información (social, ética, medioambiental, colaboradores) por medio de un análisis de contenidos para una muestra de 55 empresas chilenas cotizadas durante el periodo 2007-2012, nuestros resultados ponen de manifiesto que la implementación de las cuatro dimensiones en su conjunto, al igual que la dimensión ética y social, influyen positivamente sobre el desempeño financiero. Mientras que un tratamiento responsable a los colaboradores impacta positivamente sobre la Reputación Corporativa medida como el crecimiento de las ventas. Así, nuestro trabajo provee nueva evidencia para una economía emergente y algunas luces acerca del efecto potencial que tiene la adopción de prácticas de RSE.

PALABRAS CLAVE | Responsabilidad social empresarial, reputación corporativa, desempeño financiero, dimensión ética, dimensión colaboradores.

\section{RESUMO}

Este trabalho busca verificar se a adoção de práticas de divulgação voluntária da Responsabilidade Social Corporativa (RSC) por vários canais de comunicação tem influência no desempenho financeiro e na reputação corporativa. Foram identificadas quatro dimensões da RSC (social, ética, ambiental e parcerias) usando uma amostra de 55 empresas chilenas no período de 2007-2012. Nossos resultados mostram a adoção das quatro dimensões de RSC simultaneamente: as dimensões social e ética melhoram o desempenho financeiro, ao passo que uma relação sólida com os parceiros melhora a reputação corporativa. Assim, fornecemos novas evidências para a economia dos mercados emergentes sobre a importância da adoção de estratégias de RSC.

Ivalenzuela@unegocios.cl

Profesora de la Universidad de Chile, Facultad de Economía y Negocios -

Santiago, Chile

\section{MAURICIO JARA-BERTIN}

mjara@unegocios.cl

Profesor de la Universidad de Chile,

Facultad de Economía y Negocios -

Santiago, Chile

\section{FRANCISCO VILLEGAS PINEAUR}

fvillegas@fen.uchile.cl

Máster en Marketing por la

Universidad de Chile, Facultad de

Economía y Negócios - Santiago, Chile

PALAVRAS-CHAVES / Responsabilidade social corporativa, reputação corporativa, desempenho financeiro, dimensão ética, dimensão de parcerias.

\section{ABSTRACT \\ We analyze whether the adoption of voluntary disclosures practices on Corporate Social Responsibility (CSR) by several communicational channels has influence over financial performance and corporate reputation. Once we identify four CSR dimensions (social, ethical, environmental, partners) using a sample of 55 Chilean traded firms for the period 2007-2012. Our results show that the adoption of the four CSR dimension at the same time, and the social and ethical dimension improves financial perfor- mance, while a strength relationship with partners improve Corporate Reputation. Thus, we provide new evidence for an emerging market economy about the importance of CSR strategies adoption.}

KEYWORDS / Corporate social responsibility, corporate reputation, financial performance, ethical dimension, partners dimension. 


\section{INTRODUCCIÓN}

La Responsabilidad Social Empresarial (RSE) es un tema que ha cobrado especial importancia en las últimas décadas. Dicha importancia se ha visto plasmada, en un contexto internacional, por la proliferación de normativas e índices cuyo objetivo fundamental es establecer patrones en donde las empresas divulguen información respecto a las prácticas en temas relacionados a la RSE. Por ejemplo, el año 2010 se promulgó la normativa ISO 26.000, que guía la responsabilidad social en materias de rendición de cuentas, transparencia y comportamiento general. De manera similar, el Carbon Disclosure Project es una iniciativa que ha ganado especial relevancia en un contexto ambiental (Sullivan \& Gouldson, 2012), en donde las empresas, de una manera voluntaria aportan información cuyo resultado es la elaboración de un índice de sustentabilidad.

Nuestro trabajo, común a la preocupación internacional, intenta medir cómo influirían sobre la reputación y sobre los resultados financieros para una muestra de empresas chilenas, algunas estrategias de divulgación voluntaria de información relacionada a prácticas de RSE En el último tiempo las empresas chilenas se han acogido a la normativa ISO 26.000. No obstante, pese a los cambios que han implementado las empresas, la normativa chilena no es obligatoria respecto a qué requisitos deben cumplir en este ámbito, por lo que cualquier adopción en términos de implementación de temáticas relacionadas con RSE tiene como resultado iniciativas que recaen solamente en lo voluntario.

Considerando esta particularidad que es congruente con un enfoque holístico que muestran algunas empresas, recolectar y ordenar la información de las empresas chilenas que implementan acciones de RSE, permite generar un diagnóstico sobre la situación de las organizaciones, así como describir y caracterizar las prácticas de RSE que presentan mayor incidencia, ya que se trata de una cuestión estratégica para la gestión de los impactos sociales, económicos y medioambientales de sus operaciones.

Para el sector empresarial es fundamental contar con esta información, ya que de esta manera puede adoptar determinadas prácticas que convengan a sus intereses, involucrando a los diversos stakeholders, e incluso brindando beneficios para cada uno de ellos, en la búsqueda de un equilibrio entre los económico, lo social y lo ambiental, y por ende un impacto positivo en la empresa.

En consecuencia, resulta fundamental analizar cuáles son las principales actividades de RSE de las empresas, y las estrategias en que ponen énfasis para divulgar su información y hasta qué punto estas iniciativas de divulgación de informa- ción de prácticas en RSE influyen positiva y significativamente sobre factores medibles como por ejemplo, un incremento en el rendimiento anual de las acciones o el crecimiento anual de las ventas.

Tomando en cuenta que Chile se considera un mercado atractivo con una economía emergente, si se brinda atención a los indicadores macroeconómicos que lo reflejan tales como: el Producto Interno Bruto (PIB) que entre los años 2005 a 2011 creció a una tasa promedio anual de 4,8\% y en el 2013 mantuvo un crecimiento del $4 \%$, según cifras del Banco Central de Chile, es deseable que el crecimiento económico del sector empresarial vaya en congruencia con la filosofía de la RSE. Todo ello con la finalidad de propiciar una gestión responsable de las organizaciones en el marco del desarrollo sostenible del mundo empresarial y su entorno.

Para lograr dichos objetivos nuestro análisis se estructura en dos etapas fundamentales. En primer lugar, teniendo en cuenta cuatro dimensiones por las cuales las empresas podrían poner su énfasis en comunicación de información (ética, social, medioambiental y legal) hemos implementado un análisis de contenidos, en donde se han estudiado distintos medios tradicionales y redes sociales por los cuales las empresas emiten información. En segundo lugar, una vez identificados los énfasis en la divulgación de información, llevamos a cabo un análisis cuantitativo con la finalidad de determinar la incidencia de dichas dimensiones sobre distintos factores que aproximan el desempeño de la empresa.

Para una muestra de 55 empresas no financieras cotizadas en la Bolsa de Comercio de Santiago en el período 20072012, nuestros resultados ponen de manifiesto, en primer lugar, que dependiendo del tipo de industria, las empresas emiten información en las cuatro dimensiones: social, ética, medioambiental y colaboradores. No obstante, las dimensiones sociales y medioambientales son más transversales a todas las industrias. Por otro lado, la divulgación de información acerca de prácticas sociales y ética inciden positivamente sobre el valor de la empresa. Adicionalmente, la dimensión colaboradores resulta fundamental para tener una mejor reputación, incidiendo positivamente sobre un incremento en las ventas.

El presente trabajo se divide en seis apartados. Tras la presente introducción, en el segundo apartado se revisará los principales fundamentos de la literatura, lo que dará pie para que en un tercer apartado se proceda a la formulación de hipótesis a contrastar. La metodología, muestra y variables a utilizar será objeto del cuarto apartado, mientras que en el quinto se expondrán los principales resultados alcanzados por el estudio. Finalmente, en el sexto apartado se exponen las principales conclusiones. 


\section{FUNDAMENTACIÓN TEÓRICA}

La Responsabilidad Social Empresarial se entiende como todo proceso por el cual las empresas deciden contribuir al logro de una mejor sociedad y un medio ambiente más limpio. Esta responsabilidad se expresa frente a los empleados y, en general, hacia todos los interlocutores de la empresa y que a su vez pueden influir en su éxito (Commision-of-the-European-Communities, 2001). En un contexto general, las prácticas de RSE actúan como un agente que impulsa el crecimiento de la empresa (Aguilera \& Puerto, 2012), en especial en contextos cada vez más competitivos y dinámicos en donde, el mantenimiento y consecución de ventajas competitivas resulta cada vez más costoso.

Si bien es cierto que hay lineamientos generales en relación a RSE, su conceptualización resulta un tanto ambigua, puesto que no existe una aceptación clara y consensuada por los distintos stakeholders, lo que ha generado una diversidad de líneas de entendimiento a lo largo del tiempo. En este sentido, Rochlin (2008) argumenta que la confusión más importante acerca de la definición real de RSE radica en la asociación de este concepto a aspectos de caridad, pese a que con el tiempo ha evolucionado de manera importante, llegando a convertirse en una prioridad ineludible en las industrias más importantes de cada país (Porter \& Kramer, 2006).

Las investigaciones que han analizado el impacto de las prácticas de RSE se han centrado inicialmente en aspectos económico-financieros de las empresas (Davis, 1960). En este sentido, Gómez (2008) muestra en su revisión de literatura que la relación entre prácticas de RSE y desempeño financiero es mixta. Por ejemplo, algunos estudios han resultado entregar evidencia que apoya una relación positiva entre RSE y desempeño financiero (Fisher-Vanden \& Thorburn, 2011) y, otros estudios han mostrado lo contrario (Nelling \& Webb, 2009). Pese a la falta de consenso, es posible observar una tendencia que avala la perspectiva más difundida de manera internacional respecto de la RSE y su relación con aspectos económicos (Verduzco, 2006). De igual manera, Sierra-García, Garcia Benau y Zorio (2014) analizan las tendencias de las empresas latinoamericanas para presentar informes de RSE, y encuentran evidencia acerca del notorio incremento de esta práctica y la importancia que se está dando a nivel internacional. Por su parte, Aragon-Correa, Hurtado-Torres, Sharma y García-Morales (2008) analizan una muestra de pequeñas empresas españolas enmarcadas en el segmento de reparación automotriz y encuentran una relación positiva entre proactividad en su estrategia medioambiental y el desempeño, incluido el desempeño financiero. Resultados similares encuentran Bird, Hall, Momentè y Reggiani, (2007) desde una perspectiva de valor de la empresa.
No obstante, en estas últimas décadas ha existido un cambio de paradigma en donde no sólo los resultados financieros resultan relevantes, sino que también se destaca la importancia de contribuir en aspectos más amplios, que incluyan todos aquellos que participan en la empresa, de manera que se asegure una combinación sustentabilidad-competitividad dentro de un mundo cada día más globalizado (Barroso-Tanoira, 2008). De esta manera, se presta mayor atención al impacto de toda acción social por parte de las empresas a la comunidad (Boatright, 2000).

En este ámbito, la literatura generalmente que se ha enfocado a medir la influencia de las prácticas de RSE sobre el entorno, medido desde una perspectiva de stakeholders y de accionistas, ha identificado las dimensiones: (1) económica, (2) legal, (3) social, (4) ética y (5) medioambiental (Commision-of-the-European-Communities, 2001).

Desde una perspectiva empresarial, las prácticas de RSE también han despertado la preocupación por fortalecer la reputación corporativa. En este ámbito, Gismera y Vaquero (2000) muestran, para el contexto español, que 9 de cada 10 personas tendrían una mayor disposición a pagar productos que destinaran parte de sus ingresos a un proyecto de envergadura social, mientras que 7 de cada 10 personas lo harían por productos cuyas empresas presentan características ecológicas. En resumen, estos autores sugieren que dichos factores resultan fundamentales para fortalecer la percepción de una buena reputación empresarial, lo que podría explicar que las empresas que implementan prácticas voluntarias aceptadas por la sociedad tendrían consumidores, trabajadores y proveedores más satisfechos y alineados, lo cual potencialmente repercute significativamente sobre el rendimiento empresarial (Becker-Olsen, Cudmore, \& Hill, 2006).

Si existe un incremento en la reputación de las empresas, las empresas potencialmente gozarían de una obtención de beneficios durante períodos más prolongados, pudiendo solventar de manera más robusta cualquier circunstancia adversa, constituyéndose como un pilar fundamental para cualquier planificación estratégica en las empresas (Kotler \& Lee, 2004; Lichtenstein, Drumwright, \& Braig, 2004). No obstante, para lograr una mayor reputación corporativa las empresas deben invertir a largo plazo. De hecho, Fisher-Vanden y Thorburn (2011) muestran cómo las empresas obtienen retornos negativos anormales ante anuncios de emisión de información voluntaria acerca de adhesiones a programas de cambio climático en Estados Unidos, lo que indicaría que al mercado le toma un cierto tiempo procesar dicha información o que, la empresa se encuentra en un mal momento en términos medioambientales y su última opción es la adhesión a dichos programas. 
Por otra parte, como ya hemos mencionado, nuestro trabajo pretende llevar a cabo un análisis descriptivo basado en distintas dimensiones en el ámbito de divulgación de información voluntaria. Es por ello, que nos centraremos en una serie de modelos, como el de Carroll (1991), que sustentan dichas dimensiones, considerando que es el marco teórico más aceptado y utilizado en la literatura (Schwartz \& Carrol, 2003; Carrol \& Shabana, 2010; Homburg, Stierl, \& Bornemann, 2013).

\section{Modelos de Dimensiones de RSE}

El modelo piramidal propuesto por Carroll (1991) considera que las empresas tienen cuatro tipo de responsabilidades: (1) Responsabilidad Económica, (2) Responsabilidad Legal, (3) Responsabilidad Ética, y (4) Responsabilidad discrecional, voluntaria y filantrópica, la cual es creciente de acuerdo al nivel de compromiso que la empresa tenga con el entorno en el cual desarrolla sus actividades.

Por otra parte, Joyner y Payne (2002) extienden su análisis analizando la actitud que muestran las empresas respecto a sus prácticas de RSE y, en general, los aspectos que pueden influir en maximizar el desempeño social, lo que permite relacionar el cumplimiento de los distintos niveles de RSE propuestos por Carroll.

Finalmente, Orlitzky, Schmidt y Rynes (2003) miden distintas interrelaciones entre las variables RSE, Reputación Corporativa, Competencia, Aprendizaje, Eficiencia y Desempeño Financiero, evidenciando un efecto moderador que cumplen desde el punto de vista de la empresa, las variables internas Competencia, Aprendizaje y Eficiencia y, la variable externa Reputación Corporativa. La Figura 1 muestra cómo se relacionan dichas variables de acuerdo a los modelos analizados por la literatura (Carroll, 1991; Joyner \& Payne, 2002; Orlitzky, Schmidt, \& Rynes, 2003).

Figura 1. Modelo explicativo en RSE y su relación con la reputación corporativa y performance financiera.

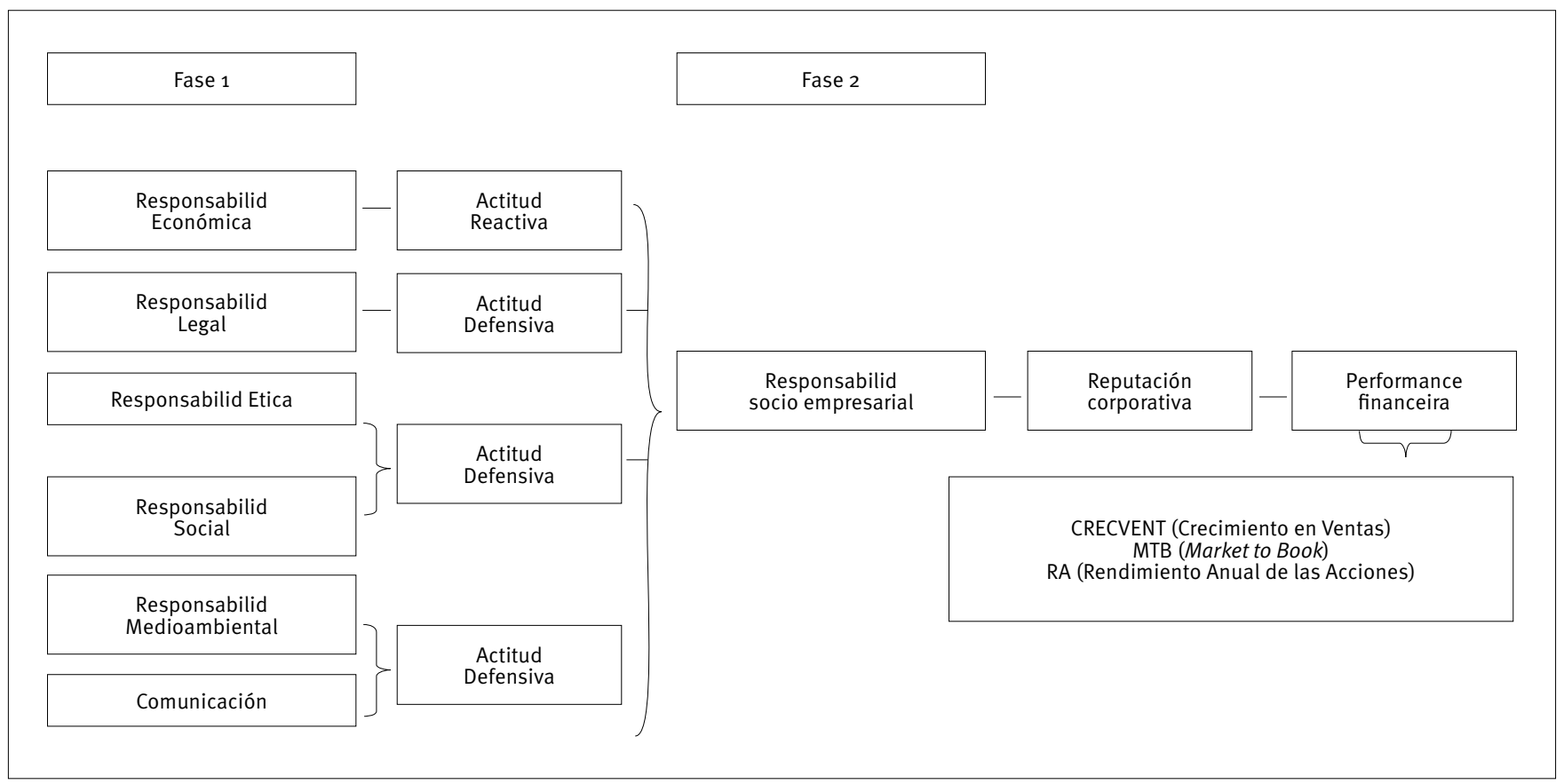

\section{FORMULACIÓN DE HIPÓTESIS}

Puesto que el objetivo fundamental de nuestro trabajo es medir cual es el efecto que tienen las prácticas de RSE y sus distintas dimensiones sobre el desempeño financiero y reputación corporativa, es que nuestras hipótesis intentarán dar respuesta ambos cuestionamientos, tanto desde una perspectiva global en donde se consideran todas las dimensiones en su conjunto, como en una perspectiva individual en donde cada dimensión se analiza de manera separada.
Como se mencionó anteriormente, estas últimas décadas la literatura que se ha centrado en el análisis de la relación entre prácticas de RSE y desempeño financiero no ha resultado ser concluyente al momento de corroborar una relación positiva (Allouche $\&$ Laroche, 2005). Adicionalmente, si las prácticas de RSE tienden a mejorar la reputación corporativa (Fombrum \& Shanley, 1990), entonces dicha mejora debiera tener resultados medibles en términos de recompensa otorgada por los consumidores en el largo plazo (Becker-Olsen, Cudmore, \& Hill, 
2006). Dado estos argumentos, es que desde una perspectiva general podemos enunciar nuestra primera doble hipótesis de investigación:

H1a: La implementación de las 4 dimensiones de la RSE tiene un efecto positivo sobre el desempeño financiero de la empresa.

H1b: La implementación de las 4 dimensiones de RSE influye positivamente sobre la Reputación Corporativa medida como el crecimiento en las ventas.

En lo tocante a la dimensión social, cabe señalar que esta ha sido uno de los ejes que ha tenido mayor relevancia desde la génesis del concepto de RSE. Esto se centra en el hecho de que todo efecto de cualquier adopción de prácticas en RSE tiene un efecto directo en la comunidad y en su contribución, entre otras cosas, al valor de marca, a la reputación y al reconocimiento público de empresa generadora de valor permanente (Villafañe, 2009). Las investigaciones realizadas por Callan y Thomas (2009) así como por Simpson y Kohers (2002), tienen como principales resultados, la existencia de una relación positiva entre la responsabilidad social y el rendimiento de la empresa. Si esto se cumple, podemos argumentar que:

H2a: La divulgación de información de prácticas de RSE en su dimensión social influye positivamente sobre el desempeño financiero de las empresas.

$\mathrm{H} 2$ b: La divulgación de información de prácticas de RSE en su dimensión social influye positivamente sobre la Reputación Corporativa medida como el crecimiento de las ventas.

Ahora bien, eventos recientes como los casos de Enron y Parmalat en un contexto internacional y la Polar en Chile (el cual es un claro ejemplo de cómo una empresa de retail ha engañado sistemáticamente al mercado a través de manipulaciones contables que decantaron en uno de los fraudes más emblemáticos en Chile), han desatado una crisis de desconfianza que ha azotado el entorno corporativo, y han traído consigo que las empresas tengan una especial preocupación por reforzar la calidad de la información que emiten al mercado y, en especial, la importancia de implementar y divulgar información relacionada con el comportamiento ético de las empresas no tiene más incentivo que incidir sobre la percepción de credibilidad. En esta línea, Salgado y Hernández (2007) evidencian para una muestra de empresas mexicanas que aquellas empresas que han emitido información acerca de prácticas éticas mostraron un mejor desempeño en comparación con aquellas empresas que no lo hicieron. Dados estos argumentos, es posible argumentar que:
H3a: La divulgación de información de prácticas de RSE en su dimensión ética influye positivamente sobre el desempeño financiero de las empresas.

H3b: La divulgación de información de prácticas de RSE en su dimensión ética influye positivamente sobre la Reputación Corporativa medida como el crecimiento de las ventas.

Por su parte, las prácticas de RSE bajo una dimensión medioambiental atienden a la preocupación de las empresas por atenuar ciertas externalidades negativas, entregando ciertas señales al mercado y la comunidad. En esta línea argumental, Boyd (2001) destaca la importancia de la sustentabilidad ambiental como una herramienta para la obtención de beneficios económicos, argumentando que las empresas que adoptan los principios de desarrollo sustentable pueden convertirse en buenos negocios. En el mismo sentido la investigación realizada por Rodríguez y Cruz (2007) arroja resultados que confirman que aquellas empresas con mayores niveles de responsabilidad social ambiental apuntan a diferenciales positivos de rentabilidad. Por lo tanto, las hipótesis planteadas bajo esta dimensión son las siguientes:

H4a: La divulgación de información de prácticas de RSE en su dimensión medioambiental influye positivamente sobre el desempeño financiero de las empresas.

H4b: La divulgación de información de prácticas de RSE en su dimensión medioambiental influye positivamente sobre la Reputación Corporativa medida como el crecimiento de las ventas.

El fortalecer los lazos con los stakeholders constituye una pieza clave en cualquier estructura de gobierno de la empresa, puesto que estos y, en especial los colaboradores, son los que cumplen un rol activo en la capacidad productiva y en la eficiencia de cualquier empresa. En esta línea, Chakravorti (2010) argumenta que los colaboradores son relevantes en la generación de valor, siendo parte fundamental en la imagen de la empresa, por lo que se les debe prestar atención implementando ciertas estrategias de RSE focalizadas, de manera de que sus intereses estén alineados a los de la empresa. En este sentido, es que podemos formular las siguientes hipótesis:

H5a: La divulgación de información de prácticas de RSE en su dimensión colaboradores influye positivamente sobre el desempeño financiero de las empresas.

H5b: La divulgación de información de prácticas de RSE en su dimensión colaboradores influye positivamente sobre la Reputación Corporativa medida como el crecimiento de las ventas.

Finalmente, el canal por donde se comunica la información hacia clientes y mercado resulta igualmente interesante, 
en especial cuando se utilizan medios no tradicionales. En este sentido, Madinabeitia (2010) plantea que las redes sociales no son canales de información sino que colocan al consumidor en el centro de la relación con la marca, siendo una parte clave del éxito empresarial. Por lo tanto, es fundamental la relevancia de las redes sociales como forma de aumentar el impacto de las acciones de RSE en el consumidor.

H6a: La divulgación de información de prácticas de RSE a través de medios no tradicionales (redes sociales) influye positivamente sobre el desempeño financiero de las empresas.

H6b: La divulgación de información de prácticas de RSE a través de medios no tradicionales (redes sociales) influye positivamente sobre la Reputación Corporativa medida como el crecimiento de las ventas.

\section{MUESTRA, VARIABLES Y METODOLOGÍA}

\section{Muestra}

La muestra empleada para nuestro contraste de hipótesis es formada por un panel desequilibrado de 323 observaciones correspondientes a 55 empresas no financieras que han cotizado en la Bolsa de Comercio de Santiago para el periodo 2007-2012. Cabe señalar que el panel es no balanceado ya que algunas empresas que pertenecen a la muestra no se encontraban cotizadas en los primeros años del periodo de estudio. Los principales sectores industriales son Alimentos y Bebidas (12,73\%), Comercio $(12,73 \%)$ y Agro y Pesca $(10,9 \%)$. Una descripción más detallada de la composición de la muestra se puede encontrar en la Tabla 1 , en donde hemos utilizado la clasificación sectorial entregada por la Superintendencia de Valores y Seguros (SVS).

La información analizada es el resultado de la conjugación de dos fuentes de información. Por un lado, la base de datos Datastream nos provee información financiera confiable acerca de la empresa. Por otro lado, a través de las Memorias Anuales, sitios web, medios de comunicación y redes sociales para cada empresa, se ha llevado a cabo una revisión que se basa en un análisis de contenidos que tiene como objetivo recolectar y ordenar la información para así describir y caracterizar las distintas acciones estratégicas en el ámbito de la RSE que implementan las empresas. Específicamente, lo que se busca es encontrar respuestas relacionadas con el contenido de estas acciones, de qué se está hablando, cuáles son las temáticas que aborda, cuáles son los principales canales de comunicación, entre otras. Cabe señalar, que el resultado de esta revi- sión ha supuesto ser la más restrictiva en términos de limitar el tamaño de la muestra.

\section{Tabla 1. Distribución sectorial de la muestra}

\begin{tabular}{|c|c|}
\hline Sectores & Observaciones \\
\hline Agro y pesca & 36 \\
\hline Alimentos y bebidas & 42 \\
\hline Comercio & 42 \\
\hline Construcción & 12 \\
\hline Energía eléctrica & 6 \\
\hline Minerales no metálicos & 24 \\
\hline Minería & 6 \\
\hline Otros & 42 \\
\hline Papel y celulosa & 6 \\
\hline Petróleo y gas & 12 \\
\hline Química & 18 \\
\hline Siderurgia y metalurgia & 17 \\
\hline Telecomunicaciones & 18 \\
\hline Transporte y servicios & 12 \\
\hline TOTAL & 323 \\
\hline
\end{tabular}

\section{Variables y Metodología}

Dado que nuestro objetivo es analizar el efecto que tienen las distintas estrategias de divulgación de información voluntaria de prácticas de RSE sobre los resultados financieros y la reputación corporativa, es que nos centraremos en tres medidas, dos relacionadas con el valor de mercado de los títulos como proxy de desempeño financiero y una variable basada en las ventas como variable que aproxima la reputación corporativa.

La literatura financiera ha definido una serie de variables que aproximan el desempeño financiero (Dechow, 1994; Anderson \& Reeb, 2003; Adam \& Goyal, 2008; Jara-Bertin \& López-Iturriaga, 2011), y que han sido empleadas en estudios relacionados al efecto que tienen las prácticas de responsabilidad social empresarial sobre el desempeño (Bird et al., 2007; Callan \& 
Thomas, 2009; Kim \& Kim, 2014). En este sentido, hemos definido como variables que aproximan el desempeño financiero la Rentabilidad anual de las acciones (RA), la cual se calcula como el incremento (decremento) de los precios de las acciones respecto al período anterior, y el market-to-book ratio (MTB), que viene estimado como la razón entre el valor de mercado del patrimonio y su valor en libros. De igual manera, en concordancia con trabajos que han evaluado las prácticas de responsabilidad social empresarial y la reputación corporativa desde una dimensión de resultados (Marín \& Ruiz, 2008; Kemper Schilke, Reimann, Wang y Brettel, 2013), es que hemos definido el crecimiento en las ventas respecto al año anterior (CRECVENT) bajo el supuesto de que toda mejora que tenga la empresa en términos de su reputación, cualquiera sea su mercado objetivo, trae consigo una mejora en las ventas. Finalmente, existe una variedad de estudios en donde se ha analizado la incidencia positiva de las prácticas de RSE sobre el rendimiento financiero de la empresa (Johnson \& Greening, 1999; Orlitzky, Schmidt, \& Rynes, 2003; Peloza, 2006; Wu, 2006)

Ahora bien, nuestras variables explicativas, que han sido el resultado del análisis de contenido, se centran en la identificación de las distintas dimensiones por las cuales las empresas han emitido información voluntaria acerca de prácticas en RSE. En este sentido, hemos definido 4DIM como una variable dicotómica que toma el valor 1 cuando las empresas han divulgado información a través de las cuatro dimensiones identificadas en su conjunto y cero en otro caso. Por su parte, DSOCIAL es una variable dicotómica que toma el valor 1 cuando la empresa ha emitido información voluntaria acerca de prácticas de RSE en su dimensión social y cero en otro caso, DETICA es una variable dicotómica que toma el valor 1 cuando la empresa ha emitido información voluntaria acerca de prácticas de RSE en su dimensión ética y cero en otro caso, DAMB es una variable dicotómica que toma el valor 1 cuando la empresa ha emitido información voluntaria acerca de prácticas de RSE en su dimensión ambiental y cero en otro caso, y DCOLAB es una variable dicotómica que toma el valor 1 cuando la empresa ha emitido información voluntaria acerca de prácticas de RSE en su dimensión colaboradores y cero en otro caso. Adicionalmente, hemos definido DREDES que es una variable que toma el valor 1 cuando el canal por el cual se emite la información es un canal que se encuentra dentro de la categoría de "redes sociales" y cero en otro caso.
De acuerdo a la literatura financiera, hemos introducido en nuestros modelos estimados una serie de variables de control que potencialmente incidan sobre el valor en la empresa (Berger \& Ofek, 1995; Campa \& Kedia, 2002; Jara-Bertin, López-Iturriaga, \& López-de-Foronda, 2008), como lo son el tamaño de la empresa (LNTAB) medido por logaritmo natural del total de activos, el grado de endeudamiento (LEV) medido por la razón de endeudamiento total sobre el total de activos, la rentabilidad sobre los activos (ROA), diversificación medida como una variable dicotómica (DIV) que toma el valor 1 cuando la empresa se encuentra diversificada y cero en otro caso, y las oportunidades de crecimiento (CAPEXSAL) estimada como el CAPEX sobre las ventas. Cabe destacar, que algunas de estas variables de control han sido utilizadas en estudios previos (Bird et al., 2007; Callan \& Thomas, 2009).

Adicionalmente, si analizamos que otros factores pueden incidir sobre el valor de la empresa, debemos tener presente algunas características que componen el sistema corporativo chileno. En este sentido, Chile representa una economía emergente enmarcado dentro de un sistema civil francés (La Porta, Lopez-de-Silanes, \& Shleifer, 1999; Lefort \& Walker, 2000; Demirgüç-Kunt \& Maksimovic, 2002; Lefort \& González, 2008), que presenta una protección al inversor externo más débil, en comparación con países de otros entornos como Estados Unidos, y presenta una marcada concentración de la propiedad, en donde las decisiones de las empresas dependen principalmente de un accionista de referencia o controlador, el que puede tener una incidencia directa sobre decisiones que maximicen valor. En este sentido, hemos introducido como variable de control a $\mathrm{P}_{1}$, que representa el porcentaje de propiedad que posee el principal accionista.

Adicionalmente, hemos introducido un conjunto de variables dicotómicas sectoriales de acuerdo a la clasificación sectorial entregada por la Superintendencia de Valores y Seguros (dummysectorial) y un conjunto de variables dicotómicas temporales (dummyaños).

Siguiendo la línea de trabajo de investigaciones previas que han relacionado el desempeño con prácticas de RSE (Bird et al., 2007; Callan \& Thomas, 2009), se procederá a estimar la ecuación (1) a través de regresiones empleando la metodología de datos de panel, en donde DX representa las distintas dimensiones definidas con anterioridad, las cuales son introducidas una a la vez.

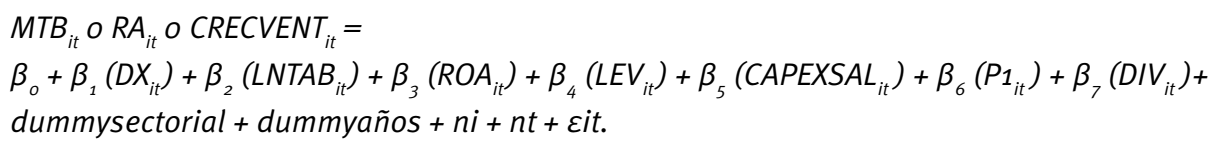


La utilización de la metodología de datos de panel se justifica por la muestra utilizada, la cual combina series de tiempo por seis años y datos de sección cruzada para 55 empresas, lo que nos permite hacer un uso más óptimo de dicha metodología (Arellano, 2003). Este procedimiento de estimación nos entrega ventajas que se derivan de controlar algunos problemas relacionados a la heterogeneidad constante e inobservable (Arellano \& Bover, 1990), esto es, algunas características específicas de cada empresa que permanecen constantes a través del tiempo, y que permanecerán constantes y representadas como un término de efectos fijos. Dado que es inobservable, se vuelven parte de un componente aleatorio del modelo estimado. En este sentido, la especificación de este tipo de relación entre el término de efectos fijos y las variables explicativas se vuelve crucial puesto que la misma relación existiría entre la variable independiente y el componente aleatorio.

Si dicha correlación existe, entonces los estimadores se tornarían inconsistentes a menos que el término de efectos fijos sea eliminado. En este sentido, las estimación intragrupos, que remueve el término de efectos fijos, provee estimadores consistentes. Consistente con dicho planteamiento, en todos los paneles de la Tabla 4 se presenta la prueba de Hausman, que es empleada para testear la hipótesis nula de ausen- cia de correlación entre las variables independientes y el término de efectos fijos.

\section{RESULTADOS}

\section{Análisis Descriptivo}

Una vez identificadas las principales dimensiones de RSE por las cuales las empresas ponen su énfasis en términos de divulgación de información voluntaria a los distintos stakeholders, la siguiente etapa de análisis corresponde al análisis descriptivo de las principales variables. En la Tabla 2 se presenta el promedio, desviación estándar, valores mínimos, máximos y de cada cuartil para cada una de las variables de estudio.

En adición, de manera de anticipar algunas relaciones y encontrar diferencias significativas entre aquellas empresas que han implementado dimensiones de estrategias de RSE y aquellas que no las han implementado, es que en la Tabla 3 se han estimado, para cada una de las dimensiones de RSE, la prueba no paramétrica de Kruskal-Wallis en donde comparamos las variables en las cuales se centran nuestras hipótesis (RA, MTB y CRECVENT).

Tabla 2. Estadísticas descriptivas

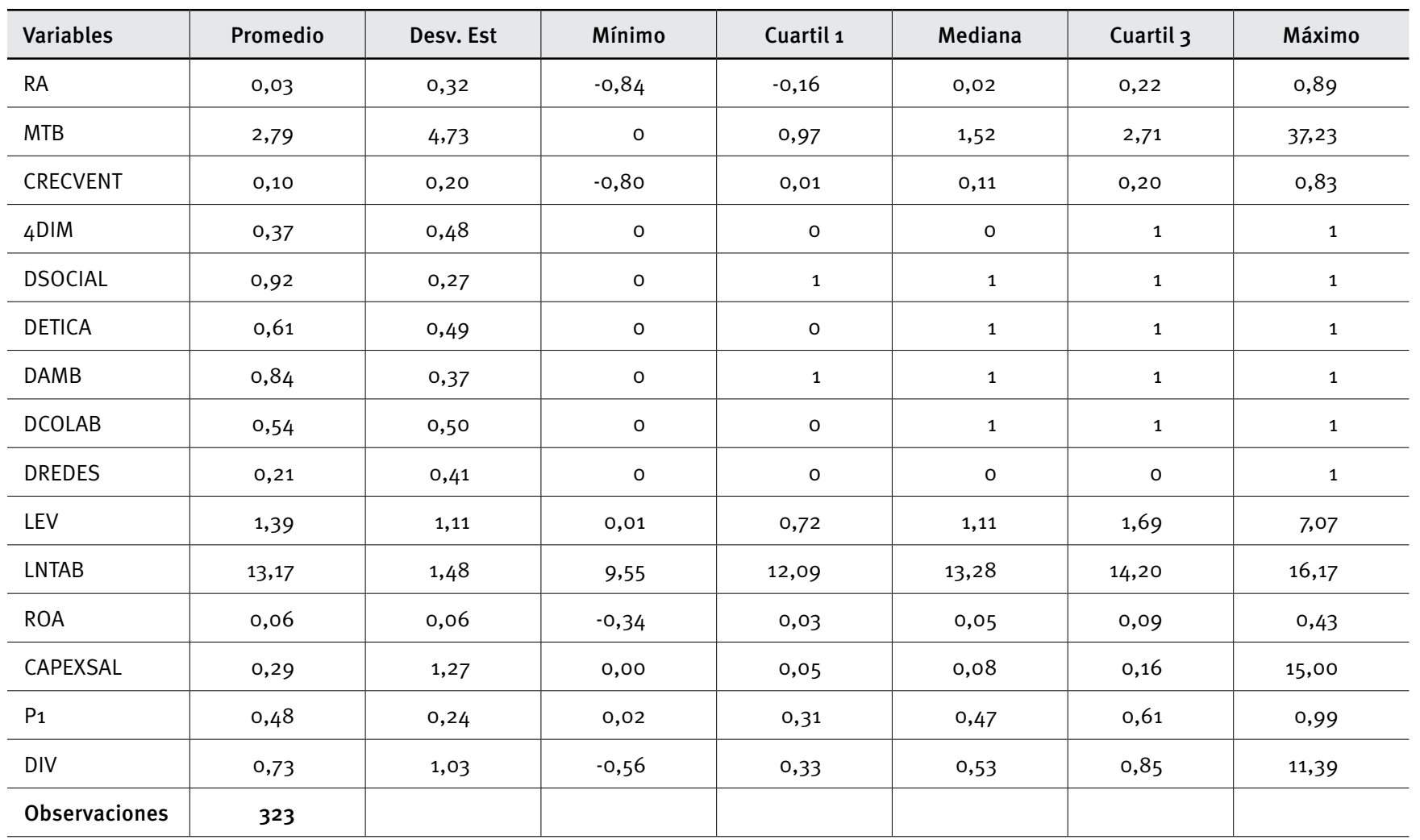


El Cuadro 2 muestra las estadísticas descriptivas para toda la muestra. El promedio de la rentabilidad anual de las acciones -Ra- es de -0,03 (0,02) y la desviación estándar es de 0,32. Asimismo, los rangos intercuartílicos van de -0,16 a 0,22. En general las empresas de la muestra presentan una buena valoración u oportunidades de crecimiento, ya que la variable MTB presenta una media (mediana) de $2,79(1,52)$ y una desviación estándar de $(2,73)$. Respecto al crecimiento de las ventas (CRECVENT), el promedio es de 0,10 y la desviación estándar de 0,20, de la misma forma los rangos intercuartílicos van de 0,01 a 0,20, lo cual es evidencia de la expansión del consumo estos últimos años en Chile en el sector del comercio principalmente.

Tabla 3. Pruebas no paramétricas de Kruskal-Wallis para las dimensiones de estudio

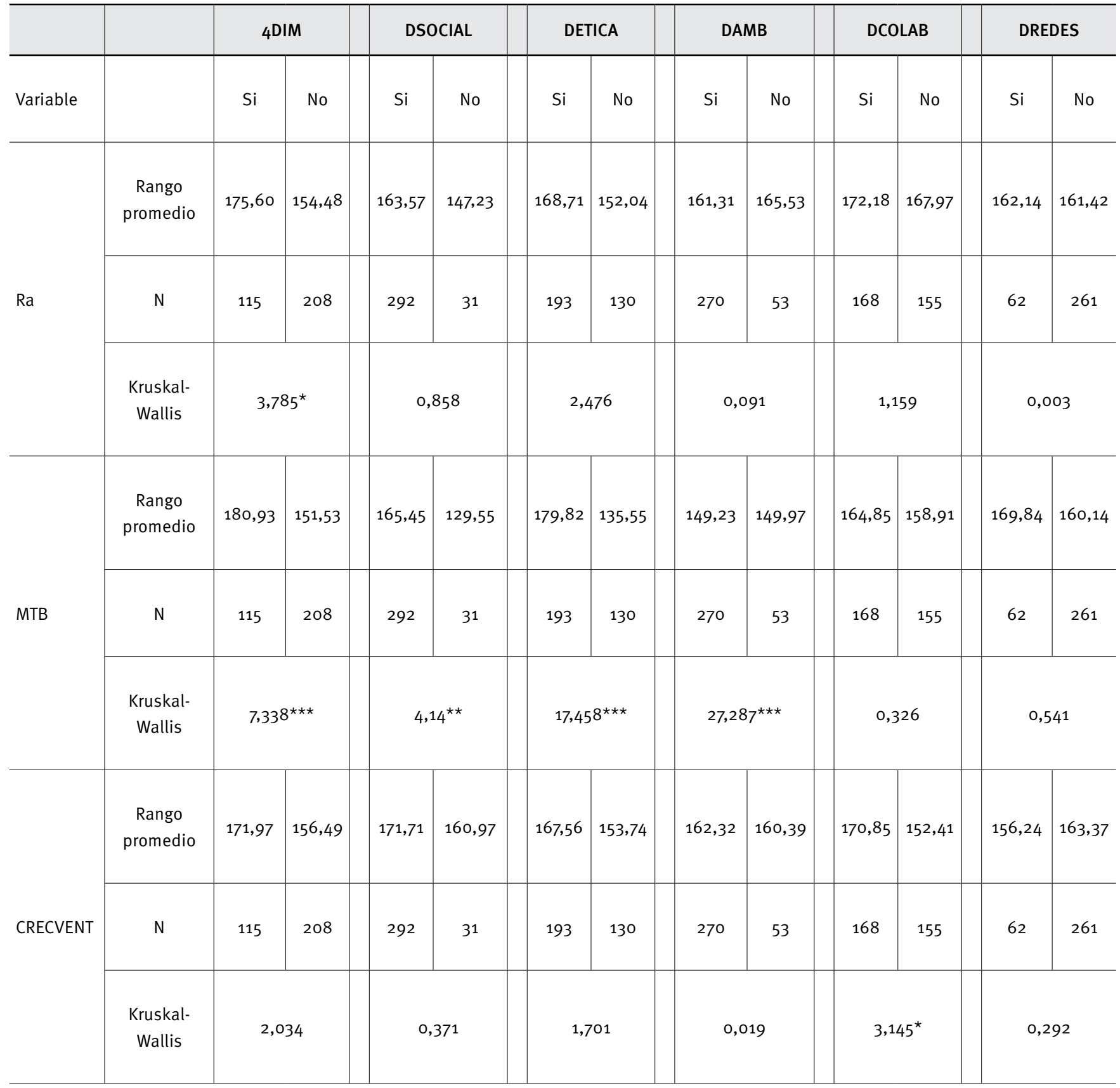

Nota: Parámetros estimados para las pruebas no paramétricas de Kruskal-Wallis, la cual tiene por hipótesis nula la ausencia de diferencias significativas entre las medidas a comparar, y se contrasta por medio de una x2. RA representa la rentabilidad anual de las acciones, MTB es el ratio valor de mercado sobre valor libro y CRECVENT es el crecimiento de las ventas desde el periodo t-1 a t. Las variables de agrupación son 4DIM, DSOCIAL, DETICA, DAMB, DCOLAB y DREDES, y toman el valor 1 cuando las empresas han emitido información en las cuatro dimensiones propuestas, en la dimensión social, ética, ambiental, colaboradores y el canal ha sido a través de redes sociales, respectivamente. ${ }^{* \star *}$ indica un nivel de significancia inferior al $1 \%,{ }^{* *}$ inferior al $5 \% \mathrm{y}$ * inferior al $10 \%$. 
En relación a las estrategias de RSE implementadas y divulgadas por las empresas, es posible observar que en promedio el $37 \%$ de las empresas han divulgado información relacionada a las 4 dimensiones de análisis. De una manera más específica, el $92 \%$ ha emitido información acerca de prácticas en la dimensión social, el $84 \%$ de las observaciones de la muestra ha implementado la dimensión medioambiental, el $61 \%$ ha destacado la importancia de divulgar prácticas relacionadas a la dimensión ética, mientras que el $54 \%$ ha reportado acciones cuya finalidad es fortalecer relaciones con sus colaboradores. Un elemento destacable es que solo un $21 \%$ de las observaciones de la muestra han comunicado sus estrategias y acciones de RSE a través de las redes sociales.

Un factor importante a destacar es la alta concentración de la propiedad observada de las empresas de la muestra. Como se puede observar cuadro 2, el promedio de la participación de la propiedad del principal accionista es de $48 \%$ (47\%) y sus rangos intercuartílicos van de $31 \%$ a $61 \%$, lo que indica que en la muestra total existe una predominancia de estructuras accionariales concentradas donde probablemente existe una clara predominancia en la toma de decisiones de un accionista controlador.

Por otra parte, como se puede apreciar en el Cuadro 3, las empresas que han implementado las cuatro dimensiones (4DIM) en general muestran diferencias significativas que apuntan a un mayor desempeño financiero y valoración (RA y MTB). De manera similar, la implementación de la dimensión social nos sugiere la existencia de diferencias significativas en términos de valoración de la empresa (MTB). En relación a la dimensión ética, esta parece ser bien recogida por los inversores en términos de valor (MTB), no así de rentabilidades de las acciones. Finalmente, el implementar la dimensión colaboradores (DCOLAB) se encuentra relacionado a un mayor nivel de crecimiento en las ventas (CRECVENT), lo que indica que dicha dimensión parece ser fundamental al momento de obtener un mejor posicionamiento en el mercado y de la reputación corporativa.

\section{Análisis empírico}

A continuación procedemos a interpretar los resultados obtenidos en las distintas estimaciones con la finalidad de contrastar los objetivos propuestos en el trabajo. Con este fin, en el Panel A y B del Cuadro 4 se muestran los resultados de las estimaciones de la ecuación (1) en donde se analiza la incidencia sobre el valor de las estrategias de divulgación de información voluntaria acerca de prácticas de RSE. De manera similar, en el Panel C de la Tabla 4 se muestran los resultados de las estimaciones de la ecuación (1) en donde se analiza la incidencia de dichas prácticas sobre la reputación corporativa medida como el crecimiento de las ventas.
Como se puede observar en la columna (A) del Panel A de Cuadro 4 , los resultados obtenidos muestran la existencia de una relación positiva y estadísticamente significativa entre la implementación de estrategias de divulgación de información voluntaria en las cuatro dimensiones de RSE en su conjunto (4DIM) y la rentabilidad anual de las acciones (RA). De manera similar, la columna (A) del Panel B del Cuadro 4 pone de manifiesto la existencia de una relación positiva y estadísticamente significativa entre las cuatro dimensiones (4DIM) y el ratio market-to-book. Estos resultados dan soporte a la hipótesis $\mathrm{H}_{1} \mathrm{~A}$ la cual sugiere que las prácticas de RSE pueden representar señales creíbles al mercado de capitales sobre del comportamiento de la empresa. No obstante, debemos tener especial cuidado al interpretar dichos resultados, puesto que potencialmente aquellas empresas que implementen las cuatro dimensiones en términos de divulgación de información voluntaria probablemente también tendrán mayores niveles de inversión y oportunidades de crecimiento, por lo que puede quedar limitado a empresas más grandes. En este sentido, empresas más grandes también tendrán mayor visibilidad al mercado y valor, por lo que las prácticas de RSE puede venir de una relación endógena (Zamagni, 2005).

Al desglosar las distintas dimensiones y medir el efecto que tiene cada una de ellas sobre el desempeño financiero, hemos encontrado que tanto la dimensión social como la dimensión ética resulta relevante al momento de explicar tanto la rentabilidad (RA) como la valoración de la empresa (MTB), lo que resulta coherente con la hipótesis $2 \mathrm{~A}$ y $3 \mathrm{~A}$. Como se puede observar en la columna (C) del panel A y B del Cuadro 4, se ha encontrado una relación positiva y significativa entre DETICA y las variables RA y MTB, respectivamente. Como se puede observar en la columna (B) del panel A y B del Cuadro 4, se ha encontrado una relación positiva y significativa entre DSOCIAL y las variables RA y MTB, respectivamente. De manera análoga, como se puede observar en la columna (C) del panel A y B del Cuadro 4 , se ha encontrado una relación positiva y significativa entre DETICA y las variables RA y MTB, respectivamente.

Estos resultados ponen de manifiesto la importancia de destacar el comportamiento social y ético de las empresas en el mercado, especialmente si se consideran algunos hechos recientes en el ámbito del gobierno corporativo que han sacudido la opinión pública y han traído una crisis de desconfianza por parte de los inversores, ejemplos de ello son el Caso La Polar donde se quebrantó la credibilidad de los consumidores dada la malversación de las políticas de crédito (pagos y renegociaciones unilaterales) y de un engaño sistemático al mercado de capitales, el caso de FASA, en la cual la colusión de precios del ámbito farmacéutico disminuyó la confianza del mercado, entre otros. 
Tabla 4. Dimensiones de RSE y Desempeño Financiero

Panel A. Regresiones para la ecuación (1) con RA como variable dependiente

(continuación)

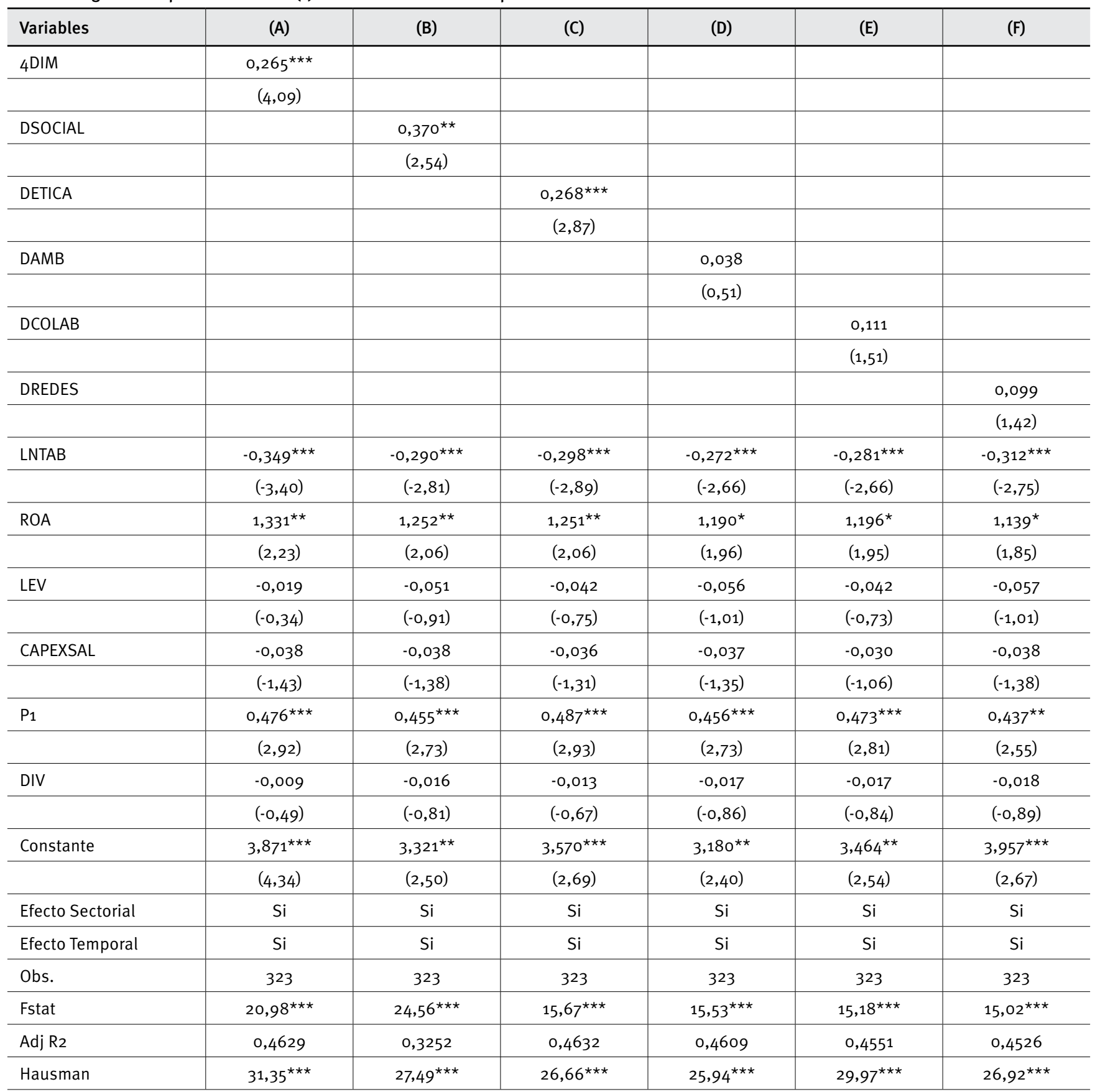

Nota: Coeficientes estimados (estadístico-t) de las estimaciones de la ecuación (1) a través de la metodología de datos de panel. En el Panel A la variable dependiente es el retorno anual de las acciones (RA), en el Panel B la variable dependiente es la razón valor de mercado sobre valor libro (MTB) y en el Panel C la variable dependiente es el crecimiento en las ventas desde t-1 a t (CRECVENT). En relación a las variables explicativos, 4DIM es una variable dicotómica que toma el valor 1 si la empresa ha divulgado información en las cuatro dimensiones propuestas en su conjunto, DSOCIAL es una variable dicotómica que toma el valor 1 si la empresa ha divulgado información voluntaria desde una perspectiva social, ETICA es una variable dicotómica que toma el valor 1 si la empresa ha divulgado información voluntaria desde una perspectiva ética, DSOCIAL es una variable dicotómica que toma el valor 1 si la empresa ha divulgado información voluntaria desde una perspectiva social, DAMB es una variable dicotómica que toma el valor 1 si la empresa ha divulgado información voluntaria desde una perspectiva ambiental, DCOLAB es una variable dicotómica que toma el valor 1 si la empresa ha divulgado información voluntaria desde una perspectiva de colaboradores, DREDES es una variable dicotómica que toma el valor 1 si la empresa ha divulgado información voluntaria a través de redes sociales, LNTAB es el logaritmo natural del total de activos, ROA es la rentabilidad sobre los activos, LEV es el apalancamiento financiero medido como el nivel de deuda sobre patrimonio, CAPEXSAL representa los gastos de capital sobre las ventas, P1 es el porcentaje en la propiedad en manos del principal accionista y DIV es la tasa de pago de dividendos. Hemos controlado todas nuestras estimaciones por efectos sectoriales y temporales. La prueba de especificación de Hausman compara los efectos fijos versus los efectos aleatorios bajo la hipótesis nula de que los efectos individuales no se encuentran correlacionados con otros regresores en el modelo y se distribuye como una x2 con tantos grados de libertad como regresores tiene el modelo. ${ }^{\star \star \star}$ indica un nivel de significancia inferior al $1 \%$, ${ }^{\star *}$ inferior al $5 \%$ y inferior al $10 \%$. 
Panel B. Regresiones para la ecuación (1) con MTB como variable dependiente

(continuación)

\begin{tabular}{|c|c|c|c|c|c|c|}
\hline Variables & (A) & (B) & (C) & (D) & (E) & (F) \\
\hline 4DIM & $2,508^{\star \star \star *}$ & & & & & \\
\hline DSOCIAL & & $3,555^{\star \star \star}$ & & & & \\
\hline \multirow[t]{2}{*}{ DETICA } & & & $1,431^{\star \star}$ & & & \\
\hline & & & $(2,51)$ & & & \\
\hline \multirow[t]{2}{*}{ DAMB } & & & & $-1,197^{\star}$ & & \\
\hline & & & & $(-1,89)$ & & \\
\hline \multirow[t]{2}{*}{ DREDES } & & & & & & 0,163 \\
\hline & & & & & & $(0,41)$ \\
\hline \multirow[t]{2}{*}{ LNTAB } & $-1,308^{\star \star \star}$ & $-1,003^{\star \star \star}$ & $-1,074^{\star \star \star}$ & $-0,688^{\star \star \star}$ & $-0,948^{\star \star \star}$ & $-1,018^{\star \star \star}$ \\
\hline & $(-4,56)$ & $(-4,82)$ & $(-4,81)$ & $(-2,84)$ & $(-3,80)$ & $(-4,92)$ \\
\hline \multirow[t]{2}{*}{ ROA } & $1,181^{*}$ & $1,014^{\star \star}$ & $1,220^{\star}$ & $1,013^{\star \star}$ & $1,149^{\star \star}$ & $1,015^{\star \star}$ \\
\hline & $(1,75)$ & $(2,04)$ & $(1,82)$ & $(2,26)$ & $(2,21)$ & $(2,32)$ \\
\hline \multirow[t]{2}{*}{$\mathrm{P}_{1}$} & $-4,335^{\star \star \star}$ & $-4,119^{\star \star \star}$ & $-4,036^{\star \star \star}$ & $-3,413^{\star \star \star}$ & $-3,786^{\star \star \star}$ & $-4,373^{\star \star \star}$ \\
\hline & $(-3,06)$ & $(-3,61)$ & $(-3,49)$ & $(-2,88)$ & $(-3,26)$ & $(-3,83)$ \\
\hline \multirow[t]{2}{*}{ DIV } & $-0,152$ & $-0,177$ & $-0,105$ & $-0,105$ & $-0,127$ & $-0,131$ \\
\hline & $(-1,09)$ & $(-0,75)$ & $(-0,44)$ & $(-0,44)$ & $(-0,53)$ & $(-0,56)$ \\
\hline \multirow[t]{2}{*}{ Constante } & $1,87^{\star \star \star}$ & $1,315^{\star \star \star}$ & $1,831^{\star \star \star}$ & $1,295^{\star \star \star}$ & $1,492^{\star \star \star}$ & $1,764^{\star \star \star}$ \\
\hline & $(4,34)$ & $(3,93)$ & $(4,88)$ & $(3,70)$ & $(4,09)$ & $(4,88)$ \\
\hline Efecto Sectorial & $\mathrm{Si}$ & $\mathrm{Si}$ & $\mathrm{Si}$ & $\mathrm{Si}$ & $\mathrm{Si}$ & $\mathrm{Si}$ \\
\hline Efecto Temporal & $\mathrm{Si}$ & Si & $\mathrm{Si}$ & $\mathrm{Si}$ & $\mathrm{Si}$ & $\mathrm{Si}$ \\
\hline Obs. & 323 & 323 & 323 & 323 & 323 & 323 \\
\hline Fstat & $21,49^{\star \star \star}$ & $21,81^{\star \star \star}$ & $22,82^{\star \star \star}$ & $22,76^{\star \star \star}$ & $24,79^{\star \star \star}$ & $23,82^{\star \star \star}$ \\
\hline Adj R2 & 0,4989 & 0,3183 & 0,3030 & 0,2921 & 0,2808 & 0,3255 \\
\hline Hausman & $32,70^{\star \star \star}$ & $20,15^{\star \star \star}$ & $19,13^{\star \star \star}$ & $22,87^{\star \star \star}$ & $23,78^{\star \star \star}$ & $22,48^{\star \star \star}$ \\
\hline
\end{tabular}

*** indica un nivel de significancia inferior al 1\% 
Panel C. Regresiones para la ecuación (1) con CRECVENT como variable dependiente

(conclusión)

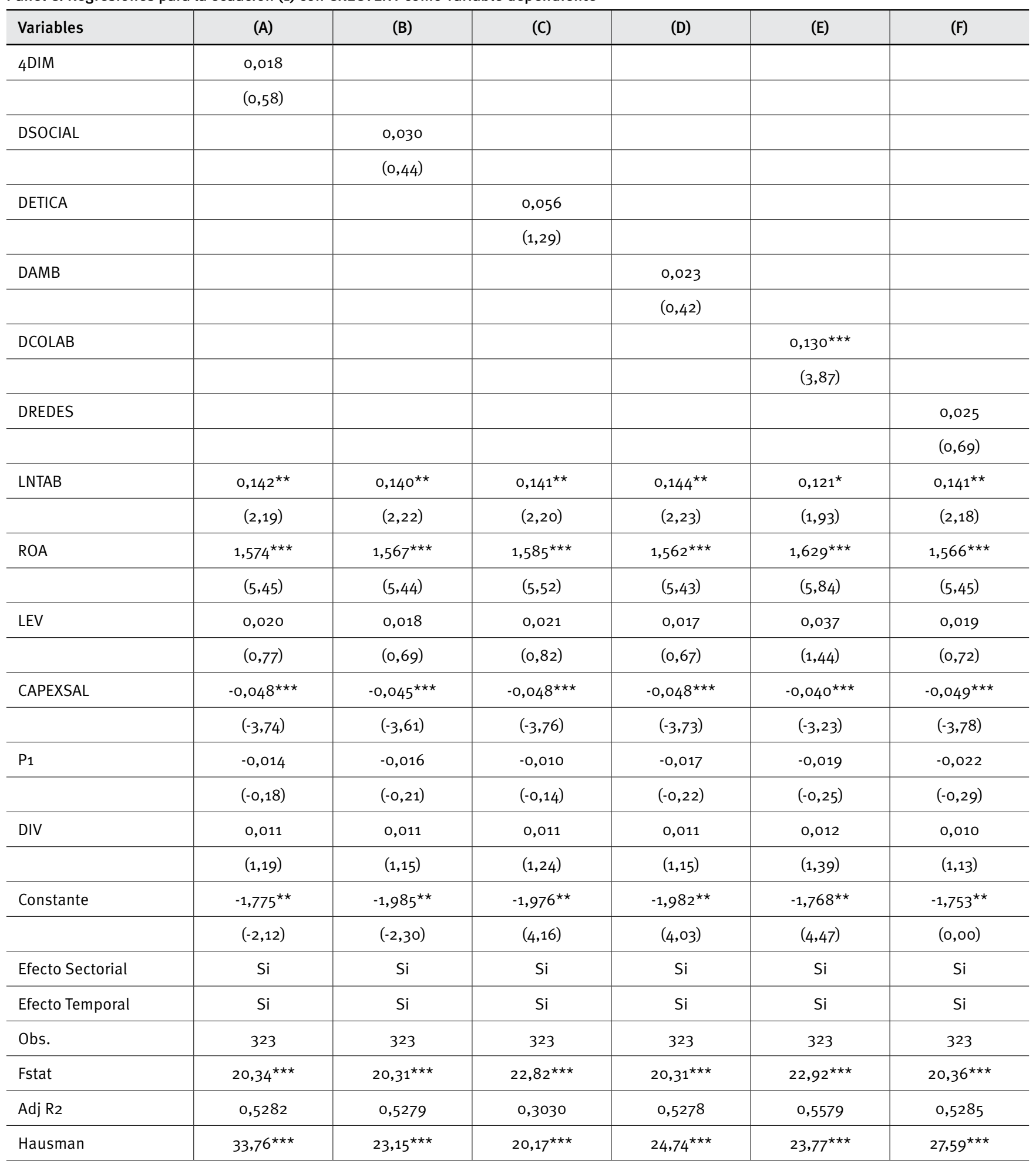

*** indica un nivel de significancia inferior al $1 \%$

En contrapartida, de la columna (D) del panel B del Cuadro 4 se observa que existe una relación negativa y estadísticamente significativa entre DAMB y MTB. Estos resultados, divergentes a los inicialmente esperados, parecen indicar que la percepción del inversor acerca de que aquellas empresas que divulgan prácticas de RSE en el ámbito ambiental, al mismo tiempo, son las empresas que presentan un mayor grado de externalidades y, por lo tanto, estas pueden ser percibidas como obligatorias. 
Finalmente, al momento de analizar qué dimensiones de divulgación de información acerca de prácticas de RSE han resultado ser relevantes, la columna (E) del Panel C del Cuadro 4 evidencia una relación positiva y estadísticamente significativa entre la implementación de estrategias de RSE orientadas hacia los colaboradores (DCOLAB) y el crecimiento en las ventas (CRECVENT) como medida aproximativa de la Reputación Corporativa. Estos resultados ponen de manifiesto la importancia de mantener una estrecha relación con los colaboradores (distribuidores, intermediarios). Además, una relación más transparente con los colaboradores permite aumentar la legitimidad de las acciones realizadas en RSE, superando las controversias que se puedan generar en el ambiente (Du \& Vieira, 2012).

\section{CONCLUSIONES}

En estos últimos años hemos visto una creciente preocupación por fortalecer y mejorar las prácticas que las empresas tienen con su entorno, de manera que la misma goce de mejores evaluaciones y de menos externalidades negativas, con la finalidad de mejorar su posición dentro de un entorno cada día más dinámico.

En Chile, esta inquietud se ha canalizado a través de distintos medios entre los que cabe destacar la adopción de normativas como la ISO 26.000 del 2010 y una serie de medidas que ha impulsado distintos entes reguladores con la finalidad de mejorar algunas prácticas relacionadas al gobierno corporativo y su comportamiento social. En un comienzo los procesos internos han sido la prioridad en los planes iniciales de RSE, como el trabajo con sus propios trabajadores. No obstante, existen muchas incógnitas acerca de cuáles prácticas de RSE podrían tener un mayor impacto en las áreas relacionadas con grupos de interés externos a la organización, como lo son los proveedores, clientes y la comunidad local.

Por consiguiente, desde la perspectiva de las implicancias empresariales, nuestro trabajo contribuye precisamente a dar respuesta a esas interrogantes estudiando la incidencia que ha supuesto la adopción de distintas prácticas de Responsabilidad Social, por parte de las empresas en Chile, sobre algunas consecuencias medibles como lo son los resultados financieros y la reputación corporativa.

Partiendo para ello, con una muestra de 55 empresas cotizadas en la Bolsa de Comercio de Santiago para el período 2007-2012, con las cuales se realiza un análisis de contenido que nos permite dilucidar qué dimensiones consideran relevantes las empresas en el ámbito de la RSE s al momento de establecer estrategias de divulgación de información. De una mane- ra más específica, nuestros resultados ponen de manifiesto la importancia de implementar las cuatro dimensiones en su conjunto y, en especial, la dimensión social y ética.

Al parecer, los recientes hechos que suponen una falta a la "ética" y han traído consigo una crisis de desconfianza en el mercado de capitales chileno, hacen visible el hecho de que los inversores pongan atención al comportamiento ético y la calidad de la información que divulgan las empresas.

Por otra parte, cabe destacar que la dimensión medioambiental tiene una influencia negativa en el valor de la empresa, lo cual tiene en consideración el hecho de que divulgar prácticas en estas áreas generan más suspicacia en el sentido de que son acciones que se asumen como obligación y compromiso de las empresas con respecto a la comunidad con la que se relacionan.

Al momento de analizar la reputación corporativa medida a través del crecimiento de las ventas, se evidencia la importancia de implementar dimensiones de RSE tendientes a fortalecer las redes con los colaboradores con la finalidad de obtener un mayor posicionamiento estratégico, mejoras en la eficiencia empresarial y en la mejor adaptación a la dinámica del entorno.

Los resultados permiten observar que es importante que las empresas implementen prácticas de RSE, ya que además del beneficio económico, posiciona a la empresa de manera estratégica con sus distintos stakeholders, lo cual permite una mayor permanencia y crecimiento en el mercado. Resultando atractivo para los inversionistas y benéfico para los consumidores.

Adicionalmente, la contribución de esta investigación para la literatura internacional en RSE radica en primer lugar, en el hecho de generar evidencia empírica en el contexto de empresas que operan en un mercado latinoamericano, con factores sociales y culturales distintos al resto del mundo. $Y$ en segundo lugar, este estudio contribuye con la propuesta de un modelo basado en la revisión de la literatura, y testeado empíricamente en este trabajo para explicar qué impactos tiene el tipo de contenido y divulgación de las estrategias de RSE y cómo influye sobre el nivel de reputación corporativa y los resultados financieros (rendimiento anual de las acciones (RA), Market to book (MTB) y crecimiento de las ventas (CRECVENT).

Este estudio podría replicarse en otros escenarios, considerando identificar un perfil similar al de las empresas objeto de estudio, así como los instrumentos de divulgación que se analizaron. Asimismo al realizar réplicas del mismo, contribuirían a identificar y ampliar la gama de estrategias de comunicación de información respecto a las prácticas de RSE utilizadas en las empresas.

Las limitaciones de este estudio subyacen en que es de carácter exploratorio a nivel de sectores empresariales, lo que 
produce una pérdida de análisis en términos de riqueza de información a nivel individual. Además es un estudio declarativo, puesto que los datos provienen de la información que las empresas publican en sus memorias, sitio web o redes sociales y consideran relevantes comunicar en sus informes a sus shareholders y stakeholders, sin poder ahondar más allá respecto a lo que efectivamente las empresas hacen, cómo lo realizan y los logros obtenidos. Por lo tanto sería interesante observar un grupo de empresas de manera aleatoria para corroborar si sus acciones y logros en RSE son consistentes con lo publicado.

\section{NOTA DE AGRADECIMIENTO}

Agradecemos los medios materiales que ha puesto a nuestra disposición el Conicyt a través de los proyectos Fondecyt de Iniciación № 11110021 y proyecto Fondecyt de Iniciación № 11100613 .

\section{REFERENCIAS}

Adam, T., \& Goyal, V. K. (2008). The investment opportunity set and its proxy variables. Journal of Financial Research, 31(1), 41-63.

Aguilera, A., \& Puerto, D. P. (2012). Crecimiento empresarial basado en la responsabilidad social. Pensamiento \& Gestión, 32, 1-26.

Allouche, J., \& Laroche, P. (2005). A meta-analytical investigation of the relationship between corporate social and financial performance. Revue de Gestion des Ressources Humaines, 57(1), 18-41.

Anderson, R., \& Reeb, D. M. (2003). Founding family ownership and firm performance: evidence from the S\&P 500., Journal of Finance, 58(3), 1301-1329.

Aragon-Correa, J. A., Hurtado-Torres, N., Sharma, S,. \& García-Morales, V. J. (2008). Environmental strategy and performance in small firms: a resource-based perspective. Journal of Environmental Management, 86(1), 88-103.

Arellano, M. (2003). Panel data econometrics Oxford: Oxford University Press.

Arellano, M., \& Bover, O. (1990). La econometría de datos de panel. Investigaciones Económicas, 14(1), 3-45.

Banco Central de Chile (2014). Ficha: Indicadores Macroeconómicos / Indicadores Coyuturales Informes Trimestrales desde el año 2005 a 2013. Recuperadp de http://www.bcentral.cl/publicaciones/estadisticas/informacion-integrada/iei13.htm en 12.05.2014.

Barroso-Tanoira, F. G. (2008). La responsabilidad social empresarial: un estudio en cuarenta empresas de la ciudad de Mérida, Yucatán. Contaduría y Administración, 226, 73-91.

Becker-Olsen, K. L., Cudmore, B. A., \& Hill, R. P. (2006). The impact of perceived corporate social responsibility on consumer behavior. Journal of Business Research, 59(1), 46-53.

Berger, P. G., \& Ofek, E. (1995). Diversification's effect on firm value. Journal of Financial Economics, 37(1), 39-65.
Bird, R., Hall, A. D., Momentè, F., \& Reggiani, F. (2007). What corporate social responsibility activities are valued by the market?. Journal of Business Ethics, 76(2), 189-206.

Boatright, R. (2000). Ethics and the Conduct of Business. 3rd ed. New Jersey: Prentice Hall.

Boyd, C. (2001). Sustainability is good business. The OECD Observer, 1., 35-37.

Callan, S. J., \& Thomas, J. M. (2009). Corporate financial performance and corporate social performance: an update and reinvestigation. Corporate Social Responsibility and Environmental Management, 16(2), 61-78.

Campa, J. M., \& Kedia, S. (2002). Explaining the diversification discount. Journal of Finance, 57( 4), 1731-1762.

Carroll, A. (1991). The pyramid of corporate social responsibility: toward the moral management of organizational stakeholders. Business Horizons, 34(4), 39-48.

Carroll, A., \& Shabana, K. (2010). The business case for corporate social responsibility: a review of concepts, research and practice. International Journal of Management Reviews, 12 (1), 85-105.

Commision-of-the-European-Communities, (2001). Green paper: promoting a European framework for corporate social responsibility. European Commision.

Chakravorti, B. (2010). Stakeholder marketing 2.0. Journal of Public Policy \& Marketing, 29(1), 97-102.

Davis, K. (1960). Can business afford to ignore corporate social responsibilities?. California Management Review, 2(3), 70-76.

Dechow, P. M. (1994). Accounting earnings and cash flows as measures of firm performance: the role of accounting accruals. Journal of Accounting and Economics, 18(1), 3-42.

Demirgüç-Kunt, A., \& Maksimovic, V. (2002). Funding growth in bankbased and market-based financial systems: evidence from firm-level data. Journal of Financial Economics, 65(3), 337-363.

Du, S., \& Vieira, E. (2012). Striving for legitimacy through corporate social responsibility: insights from oil companies. Journal of Business Ethics, 110(4), 413-427.

Fisher-Vanden, K., \& Thorburn, K. (2011). Voluntary corporate environmental initiatives and shareholder wealth. Journal of Environmental Economics and Management, 62(3), 430-445.

Fombrum, C. J., \& Shanley, M. (1990). What's in a name? Reputation building and corporate strategy. The Academy of Management Journal, 33(2),233-258.

Sierra-García, L., Garcia Benau, M. A., \& Zorio, A. (2014). Credibilidad en Latinoamérica del informe de responsabilidad social corporativa $R A E$ Revista de Administração de Empresas, 54(1), 28-38.

Gismera, L., \& Vaquero, M. (2000). La responsabilidad social de las empresas en España: la acción social. Papeles de Economía y Dirección, $5,1-8$.

Gómez, F. (2008). Responsabilidad social corporativa y performance financiero: treinta y cinco años de investigación empírica en busca de un consenso. Principios: Estudios de Economía Política, 11, 5-24.

Homburg, C., Stierl, M., \& Bornemann, T. (2013). Corporate social responsibility in business-to-business markets: how organizational customers account for supplier corporate social responsibility engagement. Journal of Marketing, 77(6), 54-72.

Jara-Bertin, M. \& López-Iturriaga, F. J. (2011). La calidad e importancia de las utilidades contables para las empresas cotizadas en los mercados de capitales chilenos. El Trimestre Económico, 78(311), 643-674. 
Jara-Bertin, M., López-Iturriaga, F. J., \& López-de-Foronda, O. (2008). The contest to the control in European family firms: how other shareholders affect firm value. Corporate Governance: An International Review, 16(3), 146-159.

Johnson, R. A., \& Greening, D. W. (1999). The effects of corporate governance and institutional ownership types on corporate social performance. Academy of Management Journal, 42 (5), 564-576.

Joyner, B., \& Payne, D. (2002). Evolution and implementation: a study of values, business ethics and corporate social responsibility. Journal of Business Ethics, 41(1), 297-311.

Kemper, J., Schilke, O., Reimann, M., Wang, X., \& Brettel, M. (2013). Competition-motivated corporate social responsibility. Journal of Business Research, 66(10), 1954-1963.

Kim, M., \& Kim, Y. (2014). Corporate social responsibility and shareholder value of restaurant firms. International Journal of Hospitality Management, 40(, 120-129.

Kotler, P., \& Lee, N. (2004). Best of breed. Stanford Social Innovation Review, 1(4), 14-23.

La Porta, R., Lopez-de-Silanes, F., \& Shleifer, A. (1999). Corporate ownership around the world. Journal of Finance, 54(2), 471-517.

Lefort, F., \& González, R. (2008). Hacia un mejor Gobierno Corporativo en Chile. Revista ABANTE, 11(1), 17-37.

Lefort, F., \& Walker, E. (2000). Ownership and capital structure of Chilean conglomerates: facts and hypotheses of governance. Revista ABANTE, 3(1), 3-27.

Lichtenstein, D. R., Drumwright, M. E., \& Braig, B. M. (2004). The effect of corporate social responsibility on customer donations to corporatesupported nonprofits. Journal of Marketing, 68(4),16-32.

Madinabeitia, E. (2010). La publicidad en medios interactivos: en busca de nuevas estrategias. Telos: Cuadernos de Comunicación e Innovación, 82, 43-54.

Marín, L., \& Ruiz, S. (2008). La evaluación de la empresa por el consumidor según sus acciones de RSC. Cuadernos de Economía y Dirección de la Empresa, 11(35), 91-112.

Nelling, E., \& Webb, E. (2009). Corporate social responsibility and financial performance: the "virtuous circle" revisited. Review Quantitative Finance and Accounting, 32(2), 197-209.
Orlitzky, M., Schmidt, F., \& Rynes, S. (2003). Corporate social and financial performance: a meta-analysis. Organization Studies, 24(3), 403-441.

Peloza, J. (2006). Using corporate social responsibility as insurance for financial performance. California Management Review, 48, 51-72.

Porter, M. E., \& Kramer, M. R. (2006). Strategy and society: the link between competitive advantage and corporate social responsibility. Harvard Business Review, 84(12), 78-92.

Rochlin, S. (2005). Llevar la responsabilidad corporativa al ADN de su empresa. Harvard Business Review, 83(8), 31-38.

Rodríguez, F. J., \& Cruz, Y. (2007). Aproximación a la incidencia de la responsabilidad social-medioambiental en el rendimiento económico de la empresa hostelera española. Revista Europea de Dirección y Economía de la Empresa, 16(1), 47-66.

Salgado, P., \& Hernández, P. (2007). La responsabilidad social en empresas del Valle de Toluca (México): un estudio exploratorio. Estudios Gerenciales, 23(102),119-135.

Schwartz, M., \& Carroll, A. (2003). Corporate social responsibility: a three-domain approach. Business Ethics Quarterly, 13(4), 503-530.

Simpson, W. G., \& Kohers, T. (2002). The link between corporate social and financial performance: evidence from the banking industry. Journal of Business Ethics, 35(2), 97-109.

Sullivan, R., \& Gouldson, A. (2012). Does voluntary carbon reporting meet investors' needs?. Journal of Cleaner Production, 36, 60-67.

Verduzco, A. (2006). Responsabilidad social empresarial: de la dimensión corporativa a la personal. The Anáhuac Journal, 6(1), 100-111.

Villafañe, J. (2009). Reputación corporativa y RSC: bases empíricas para un análisis. Telos: Cuadernos de Comunicación e Innovación, 79, $75-82$.

Wu, M. L. (2006). Corporate social performance, corporate financial performance, and firm size: a meta-analysis. Journal of American Academy of Business, 8(1), 163-171.

Zamagni, S. (2005). El fundamento ético de la responsabilidad social de la empresa. Universitas, Revista de Sociedad y Cultura de la Pontificia Universidad Católica de Argentina, 1, 2-23. 